\title{
Capacidad de resiliencia según tendencia religiosa y género en universitarios
}

\section{Resilience Capacity by Religious Tendency and Gender in University Students}

\author{
Silvia San Román Mata (*) silviasanroman@ugr.es \\ Asunción Martínez Martínez (*) asuncionmm@ugr.es \\ Félix Zurita Ortega (*) felixzo@ugr.es \\ Ramón Chacón Cuberos (*) ramonchaconcuberos@correo.ugr.es \\ Pilar Puertas Molero (*)pilarpuertasmolero@gmail.com \\ Gabriel González Valero (*) gabrielgonzalezvalero@gmail.com \\ (*) Universidad de Granada \\ (Recibido: 4 de junio de 2017; Aceptado para su publicación: 29 de agosto de 2017)
}

Cómo citar: San Román, S., Martínez, A., Zurita, F., Chacón, R., Puertas, P. y González, G. (2019). Capacidad de resiliencia según tendencia religiosa y género en universitarios. Revista Electrónica de Investigación Educativa, 21, e15, 1-11. doi:10.24320/redie.2019.21.e15.2016

\section{Resumen}

Este estudio de tipo descriptivo y corte transversal, realizado en una muestra de 597 estudiantes universitarios españoles, tiene como objetivo principal describir sus niveles de resiliencia, así como analizar la relación existente entre este constructo, el género y la tendencia religiosa. Se empleó como principal instrumento la escala de resiliencia Connor-Davidson (CD-RISC). Los resultados revelan que el género masculino muestra puntuaciones más elevadas en optimismo y adaptación a situaciones estresantes, mientras que el femenino posee una espiritualidad más elevada. Asimismo, se pudo observar que los cristianos y los ateo/agnósticos obtenían medias más elevadas en desafío de la conducta orientada a la acción, mientras que los musulmanes obtenían una mayor espiritualidad.

Palabras clave: Resiliencia, religión, género, estudiante universitario.

\section{Abstract}

The main objective of this descriptive, cross-sectional study, conducted with a sample of 597 university students from Spain, is to describe students' levels of resilience and analyze how they relate to gender and religious tendency. The main instrument used was the Connor-Davidson Resilience Scale (CD-RISC). The results show that male students exhibit higher scores in optimism and adapting to stressful situations, whereas females are more spiritual. It was also noted that Christians and atheists/agnostics obtained higher scores, on average, in defying action-oriented behavior, whereas Muslims scored more highly in spirituality.

Keywords: Resilience, religion, gender, university students. 


\section{Introducción}

La resiliencia es un concepto no reciente (Del Rincón, 2016; Kalawski y Haz, 2003), aunque es cierto que en la última década se ha utilizado en diversos contextos. De hecho, la perspectiva multidisciplinar del término se extiende a ámbitos como el educativo, social, empresarial, médico y psicológico, entre otros (Aslan y Araza, 2015; Maltby et al., 2016; Pisarska, Eisman, Ostaszewski y Zimmerman, 2016).

Atendiendo a su acepción psicosocial, este constructo podría definirse como la capacidad del ser humano para adaptarse positivamente a cualquier adversidad, resultado de una interrelación de factores de diverso tipo, constituyendo así un aprendizaje que puede potenciarse y desarrollarse (Bajaj y Pande, 2016; García del Castillo, García del Castillo-López, López-Sánchez y Dias, 2016; Hu, Zhang y Wang, 2015; Losada y Latour, 2012; Masten, 2014; Satici, 2016).

La capacidad de resiliencia se ve influenciada por diversos factores internos y externos, semejantes a la inteligencia emocional, las habilidades sociales, el contexto social en el que se desenvuelve el individuo, la familia o los amigos, entre otros (Bandura, 2011; Bravo y López, 2016; Rodríguez-Fernández, Ramos-Díaz, Ros, Fernández-Zabala y Revuelta, 2016; Villacieros, 2016). Del mismo modo, hay que señalar la importancia que supone el peso de factores que pueden actuar como protectores o disruptores en el desarrollo de dicha competencia y que en cierto modo se encuentran ligados a la calidad de vida. Entre ellos se pueden nombrar la autoestima, el autoconcepto, la asertividad, la capacidad de resolución de conflictos, los vínculos familiares o las redes de apoyo social que posea el individuo (De la Fuente et al., 2014; González, Valdez, Van Barneveld y González, 2012; Quiceno, Mateus, Cardenas, Villareal y Vinaccia, 2013; Sánchez-Teruel y Robles-Bello, 2014).

La etapa universitaria constituye un período de transición de la adolescencia a la adultez joven y se caracteriza entre otras cosas por el abandono del hogar familiar, ya que muchos jóvenes van a vivir a pisos compartidos, residencias o colegios mayores, donde se produce una modificación de las relaciones interpersonales, se generan nuevas experiencias, situaciones de peligro, vulnerabilidad y estrés, y donde la capacidad de resiliencia se encuentra presente en cierto modo (Allan, McKenna y Dominey, 2014; Beguerí, Klenzi y Malberti, 2013; Chacón et al., 2016; Hande, Börkan, Erkman y Serbest, 2016; ObradorsRial, Ariza y Muntaner, 2014).

De hecho, es imprescindible que durante esta etapa evolutiva se consoliden e instauren hábitos saludables en el joven para prevenir conductas disruptivas que puedan afectar su salud física, psíquica y emocional; la capacidad de resiliencia es un canal para ello (Álvaro, Zurita, Castro, Martínez y García, 2016; Chacón et al., 2017).

El número de investigadores que estudian la capacidad de resiliencia en jóvenes estudiantes, planteando diferencias según el género, nivel académico y origen geográfico y cultural, va en aumento (BarrantesBrais, Sánchez-Ureña y Ureña-Bonilla, 2016; Llanos, 2009; Saavedra, Castro y Saavedra, 2012; Sandoval, García-Jiménez y Pérez-Mayo, 2015; Zurita, Castro, Linares y Chacón, 2017). En este sentido, diversos trabajos de investigación alegan diferencias en la capacidad de resiliencia según el género del individuo, hallando a la mujer más resiliente que el hombre, pues ellas muestran una alta relación entre resiliencia y calidad de vida. La explicación que aportan a este hecho se debe a que el hombre se encuentra expuesto a mayores situaciones de riesgo y debilidad que la mujer (González y Valdez, 2007). Hay que tener en cuenta la acepción del término "género" en torno al cual gira este estudio, ya que éste ofrece una fuerte relación con el carácter sociocultural, que lo distingue de la connotación biológica y corporal del concepto de sexo o de diferencia sexual (De Barbieri, 1996).

Una de las características asociadas a la capacidad de superación y resiliencia es la espiritualidad, entendida desde el conjunto de creencias transcendentales sobre Dios y su existencia, y equiparándola con el término "religiosidad" (Jones, 2007; Simkin y Azzollini, 2015; Wahl, Cotton y Harrison-Monroe, 2008; Walker, Reid, O’Neill y Brown, 2009). A este respecto, durante las últimas décadas, España ha pasado de ser un país mayoritariamente católico a tener una amplia variedad de minorías religiosas debido a los procesos migratorios existentes, al turismo que atrae el país (Montes, 2016) y especialmente por la separación del Estado y la Iglesia católica (Griera, Martínez-Ariño, Clot-Garrell y García-Romeral, 
2015; Pérez-Agote 2012).

A pesar de la decadencia y caída de las creencias religiosas en términos generales y debido al proceso de modernización en que se ha visto inmersa Europa (Arroyo, 2014), autores como Rehm y Allison (2009) realizan un trabajo de investigación en el que analizan la opinión sobre la espiritualidad de alumnos universitarios, quienes la definen como un modo para conservar tanto la congruencia como la capacidad de adaptación, ya que aumenta la autoeficacia, el anhelo de obtener el potencial pleno y la responsabilidad.

Como se ha observado en la literatura existente, la capacidad de resiliencia en estudiantes universitarios ha sido estudiada en diversas situaciones; sin embargo, son escasos los trabajos de investigación que la analizan en relación con la tendencia religiosa y el género. Por ello, este estudio plantea los siguientes objetivos: 1) describir los niveles de resiliencia y factores socio-religiosos en una muestra de jóvenes universitarios españoles; 2) analizar la relación existente entre la capacidad de resiliencia, género y tendencia religiosa, y c) realizar un modelo de regresión para predecir la capacidad de resiliencia, ámbito de conocimiento y tendencia religiosa en el estudiante universitario en función del género.

\section{Método}

Se presenta un estudio no experimental, de tipo descriptivo, ex post facto y de grupo simple. Participaron 597 estudiantes universitarios de entre 19 y 23 años ( $M=23.04$ años; $D T=3.71$ ), divididos en $26.1 \%$ hombres ( $n=156)$ y $73.9 \%$ mujeres $(n=441)$. Según las áreas de conocimiento, se analizaron a 280 estudiantes de Ciencias Sociales (46.9\%) y 317 de Ciencias de la Salud (53.1\%) de la Universidad de Granada, realizando un muestreo aleatorio simple para la selección de los participantes. Como criterio de inclusión se optó porque los estudiantes estuvieran matriculados en los grados 20., 30. y 40. de los campus de Granada, Ceuta y Melilla (España). Se excluyeron del estudio 28 cuestionarios mal cumplimentados.

En el estudio se emplearon como principales instrumentos:

a) Escala de resiliencia Connor-Davidson (CD-RISC, por sus siglas en inglés) (Connor y Davidson, 2003), adaptado al español por García-Portilla et al. (2008). El instrumento se encuentra constituido por 25 ítems que se agrupan en 5 dimensiones: Locus de Control y Compromiso (LCC), conformada por la sumatoria de las cuestiones 21, 4, 19, 22 y 17; Autoeficacia y Resistencia al Malestar (ARM), formado por la suma de las cuestiones 7, 10, 16, 24, 23, 11, 15, 18 y 5; Optimismo y Adaptación a Situaciones Estresantes (OASE), según sumatorio de los ítems 1, 6, 8, 12 y 14; Desafío de la Conducta Orientada a la Acción (DCOA), conformado por la suma de los ítems 2 y 13; Espiritualidad (ES), a través del sumatorio de los ítems 3, 9 y 20. Los participantes responden a las cuestiones mediante una escala tipo Likert de cuatro opciones de respuesta donde 1 es "Nunca" y 4 es "Siempre". Para determinar las dimensiones se establece la sumatoria de los ítems referenciados anteriormente. La fiabilidad de este instrumento para la muestra estudiada fue fijada mediante el coeficiente alpha de Cronbach, obteniéndose un valor $a=.856$, considerado adecuado.

b) Cuestionario ad hoc. Se empleó un cuestionario de elaboración propia en el que se incluyeron cuestiones abiertas y cerradas que cubren aspectos sociodemográficos, como las relacionadas con el género, edad o tendencia religiosa, donde destacan, entre otras, preguntas como: ¿Profesas alguna religión?, en caso afirmativo indica cuál. ¿Eres practicante? ¿Tus padres o tutores legales practican la misma tendencia religiosa?

\subsection{Procedimiento}

Se solicitó la colaboración de los participantes mediante una carta informativa en la que se solicitaba su consentimiento. Aunque los participantes fueron informados de las características generales de la investigación, desconocían la finalidad para evitar respuestas no sinceras y reducir al máximo el efecto de deseabilidad social. Es en este punto donde se destacan las preguntas elaboradas dentro del marco de innovación docente, con la finalidad de dar respuesta a los objetivos del mismo. Después se procedió a la recogida de datos y al terminar esta actividad se explicó de forma concreta el objetivo del estudio. 
Del total de 625 participantes se eliminaron 28 cuestionarios por estar mal cumplimentados, por lo que la muestra final fue de 597. Investigadores preparados para tal efecto administraron los cuestionarios en horario no lectivo y de manera grupal. En todo momento se tuvieron en cuenta consideraciones éticas como garantizar el anonimato de la información recogida.

\subsection{Análisis de los datos}

El análisis estadístico se realizó mediante el paquete estadístico SPSS 22.0. Se usaron frecuencias y medias para los descriptivos básicos, mientras que para el estudio comparativo se emplearon t-Student y Anova en función de la naturaleza de las variables. En el modelo de regresión logística binaria para predecir los niveles de resiliencia, ámbito de conocimiento y tendencia religiosa según el género de los participantes, se empleó la prueba del chi-cuadrado $(p \leq .05)$ de la prueba de ómnibus para determinar la viabilidad del modelo. Se utilizó el R cuadrado de Cox y Snell y el R cuadrado de Nagelkerke para definir el porcentaje de la variable dependiente que predice el modelo. La forma de introducir las variables se hizo mediante selección hacia adelante condicional. El Índice de Confiabilidad (IC) para el Exp (B) fue fijado en un 95.5\% y la bondad de ajuste del modelo se comprobó mediante la prueba de Hosmer-Lemeshow.

\section{Resultados}

Los descriptivos básicos (ver tabla I) revelan que la tendencia religiosa más profesada es la cristiana con un 54.9\% ( $n=328)$, seguida de la atea-agnóstica con un $27.5 \%$ ( $n=164)$, la musulmana con un $16.2 \%$ ( $n=97$ ) y otras con un $1.3 \%(n=8)$. Sobre las dimensiones de resiliencia se observa de forma global que prácticamente todas obtuvieron una puntuación media superior a 3. La autoeficiencia y resistencia al malestar fue la dimensión más valorada $(3.23 \pm .43)$ mientras que la espiritualidad fue la que reflejó menor puntuación (2.78土.59).

Tabla I. Descriptivo del estudio

\begin{tabular}{|c|c|c|c|c|c|c|c|c|c|}
\hline \multicolumn{8}{|c|}{ Tendencia Religiosa } & & \\
\hline \multicolumn{2}{|c|}{ Cristiana } & \multicolumn{2}{|c|}{ Musulmana } & \multicolumn{2}{|c|}{ Ateo-Agnóstica } & \multicolumn{2}{|c|}{ Otras } & & \\
\hline 328 & $54.9 \%$ & 97 & $16.2 \%$ & 164 & $27.5 \%$ & 8 & $1.3 \%$ & & \\
\hline \multicolumn{10}{|c|}{ Resiliencia } \\
\hline \multicolumn{2}{|c|}{ LCC } & \multicolumn{2}{|c|}{ DCOA } & \multicolumn{2}{|c|}{ ARM } & \multicolumn{2}{|c|}{ OASE } & \multicolumn{2}{|c|}{ ES } \\
\hline M & DT & $M$ & DT & $M$ & DT & M & DT & M & DT \\
\hline 3.10 & .516 & 3.21 & .697 & 3.23 & .428 & 3.03 & .488 & 2.78 & .596 \\
\hline
\end{tabular}

En la tabla II se muestra la tendencia religiosa según el género, arrojando diferencias estadísticamente significativas $(p=.001)$. Éstas se reflejan en la tendencia cristiana, dado que más de la mitad de los participantes se adhieren a esta religión independientemente de su género. Asimismo, estas diferencias estadísticas pueden observarse en los musulmanes, donde las mujeres superan ampliamente a los hombres (19\% frente al 8.3\%).

Tabla II. Relación entre el género y la tendencia religiosa

\begin{tabular}{l|c|c|c|c}
\hline \multicolumn{4}{c}{ Tendencia Religiosa } \\
\cline { 2 - 5 } & Cristiana & Musulmana & Ateo-Agnóstica & Otras \\
\hline Hombre & $83(53.2 \%)$ & $13(8.3 \%)$ & $56(35.9 \%)$ & $4(2.6 \%)$ \\
Mujer & $245(55.6 \%)$ & $84(19.0 \%)$ & $108(24.5 \%)$ & $4(0.9 \%)$ \\
Total & $328(54.9 \%)$ & $97(16.2 \%)$ & $164(27.5 \%)$ & $8(1.3 \%)$ \\
\hline
\end{tabular}

El estudio de la capacidad de resiliencia en función del género (ver tabla III) reveló diferencias estadísticas en dos de sus dimensiones ( $p \leq .05$ ). Éstas se reflejan en el optimismo y adaptación a situaciones estresantes, donde el género masculino obtiene mayores puntuaciones medias que el femenino (3.12 \pm .48 vs 2.99 vs .48). También se observan asociaciones estadísticas para la espiritualidad, dimensión 


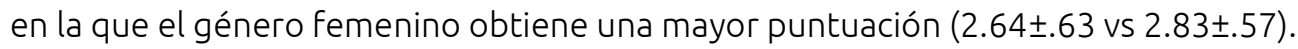

Tabla III. Relación entre el género y la resiliencia mediante una $t$ de Student

\begin{tabular}{|c|c|c|c|c|c|c|c|c|c|c|}
\hline \multirow[b]{2}{*}{ Resiliencia } & \multirow[b]{2}{*}{ Género } & \multicolumn{4}{|c|}{ Prueba de Levene } & \multicolumn{5}{|c|}{ Prueba $t$ para la igualdad de medias } \\
\hline & & M & DT & $\mathrm{F}$ & Sig. & $\mathrm{T}$ & $\mathrm{Gl}$ & Sig. & $\begin{array}{c}\text { Dif } \\
\text { medias }\end{array}$ & $\begin{array}{l}\text { Error } \\
\text { típ. }\end{array}$ \\
\hline \multirow{2}{*}{ LCC } & Hombre & 3.11 & .520 & .032 & .859 & .276 & 595 & .783 & .013 & .048 \\
\hline & Mujer & 3.10 & .515 & & & .275 & 270.00 & .784 & .013 & .048 \\
\hline \multirow{2}{*}{ DCOA } & Hombre & 3.14 & .700 & .100 & .752 & -1.325 & 595 & .186 & -.086 & .065 \\
\hline & Mujer & 3.23 & .695 & & & -1.320 & 270.20 & .188 & -.086 & .065 \\
\hline \multirow{2}{*}{ ARM } & Hombre & 3.26 & .422 & .000 & 1.000 & 1.028 & 595 & .304 & .041 & .040 \\
\hline & Mujer & 3.22 & .430 & & & 1.037 & 276.59 & .300 & .041 & .039 \\
\hline \multirow{2}{*}{ OASE } & Hombre & 3.12 & .484 & .101 & .751 & 2.836 & 595 & .005 & 128 & .045 \\
\hline & Mujer & 2.99 & .485 & & & 2.839 & 272.46 & .005 & 128 & .045 \\
\hline \multirow[b]{2}{*}{ ES } & Hombre & 2.64 & .634 & 1.002 & .317 & -3.487 & 595 & .001 & -.192 & .055 \\
\hline & Mujer & 2.83 & .574 & & & -3.325 & 250.45 & .001 & -.192 & .058 \\
\hline
\end{tabular}

En lo referente a la resiliencia y la tendencia religiosa (ver tabla IV), los resultados mostraron diferencias estadísticas en el desafío de la conducta orientada a la acción, dado que eran los musulmanes los que

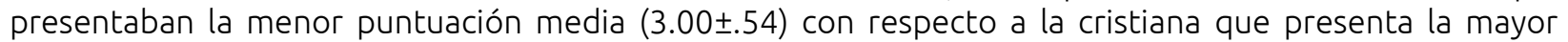
(3.26士.69). También se observa asociación para la espiritualidad, dimensión en la que la tendencia

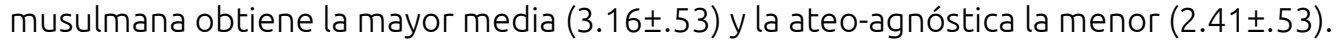

Tabla IV. Relación entre la tendencia religiosa y la resiliencia mediante la aplicación de Anova

\begin{tabular}{|c|c|c|c|c|c|}
\hline & & $M$ & DT & $\mathrm{F}$ & Sig. \\
\hline \multirow{4}{*}{ LCC } & Cristiana & 3.11 & .517 & \multirow{4}{*}{1.383} & \multirow{4}{*}{.247} \\
\hline & Musulmana & 3.00 & .541 & & \\
\hline & Ateo-Agnóstica & 3.12 & .504 & & \\
\hline & Otras & 3.13 & .260 & & \\
\hline \multirow{4}{*}{ DCOA } & Cristiana & 3.26 & .698 & \multirow{4}{*}{3.665} & \multirow{4}{*}{.012} \\
\hline & Musulmana & 3.00 & .650 & & \\
\hline & Ateo-Agnóstica & 3.23 & .699 & & \\
\hline & Otras & 3.13 & .791 & & \\
\hline \multirow{4}{*}{ ARM } & Cristiana & 3.25 & .437 & \multirow{4}{*}{.717} & \multirow{4}{*}{.542} \\
\hline & Musulmana & 3.17 & .438 & & \\
\hline & Ateo-Agnóstica & 3.23 & .402 & & \\
\hline & Otras & 3.23 & .410 & & \\
\hline \multirow{4}{*}{ OASE } & Cristiana & 3.04 & .501 & \multirow{4}{*}{.912} & \multirow{4}{*}{.435} \\
\hline & Musulmana & 2.97 & .462 & & \\
\hline & Ateo-Agnóstica & 3.04 & .480 & & \\
\hline & Otras & 2.85 & .382 & & \\
\hline \multirow{4}{*}{ ES } & Cristiana & 2.85 & .545 & \multirow{4}{*}{42.251} & \multirow{4}{*}{.000} \\
\hline & Musulmana & 3.16 & .532 & & \\
\hline & Ateo-Agnóstica & 2.41 & .534 & & \\
\hline & Otras & 2.88 & .711 & & \\
\hline
\end{tabular}

En la tabla $V$ se presentan los resultados de la regresión logística binaria para predecir el nivel de resiliencia según sus dimensiones, ámbito de conocimiento y tendencia religiosa en función del género. El modelo se ajusta bien ( $X^{2}=666.862 ; p<.001$ ) y es significativo, explica entre el 0.031 ( $R 2$ de Cox y Snell) y el 0.046 ( $R 2$ de Nagelkerke) de la variable dependiente y clasifica correctamente a un $73.9 \%$ de los participantes. Cuando se analizan de forma conjunta las variables que mejor predicen el ámbito de 
conocimiento o tendencia se observa que pertenecer al género masculino aumenta en un 1.49 estudiar Ciencias Sociales con respecto a la mujer, así como en un 1.54 el profesar una tendencia religiosa.

Tabla. V. Resultados de la regresión logística binaria para predecir los niveles de resiliencia, ámbito de conocimiento y tendencia religiosa según el género de los participantes

\begin{tabular}{l|c|c|c|c|c}
\cline { 2 - 6 } \multicolumn{1}{c|}{} & \multirow{2}{*}{ B } & \multirow{2}{*}{ Sig. } & \multirow{2}{*}{ O.R } & \multicolumn{2}{c}{ I.C. 95\% } \\
\cline { 5 - 6 } & & & & Inferior & Superior \\
\hline LCC & .112 & .728 & 1.119 & .594 & 2.105 \\
DCOA & .234 & .301 & 1.264 & .811 & 1.970 \\
ARM & -.207 & .676 & .813 & .308 & 2.148 \\
OASE & -.499 & .138 & .607 & .313 & 1.175 \\
ES & .383 & .063 & 1.466 & .980 & 2.194 \\
Ámbito de conocimiento & .405 & .034 & 1.499 & 1.031 & 2.180 \\
Tendencia religiosa & .436 & .039 & 1.546 & 1.023 & 2.336 \\
Constante & -1.497 & .000 & .224 & & \\
\hline
\end{tabular}

\section{Discusión y conclusiones}

Este trabajo se realizó con el fin de explicar la relación existente entre la capacidad de resiliencia en función de la tendencia religiosa y el género en estudiantes universitarios. Participaron alumnos universitarios de diferentes campus de la Universidad de Granada, entre ellos el de Ceuta y Melilla, ciudades transfronterizas y multiculturales, donde residen y conviven ciudadanos de las principales tendencias religiosas que existen actualmente (López-Medina, 2006).

Estudios similares atestiguan que las personas con creencias y comportamientos religiosos presentan mejores recursos y habilidades de afrontamiento y adaptación a situaciones traumáticas y adversas, manifestando una relación positiva entre religiosidad y espiritualidad con el desarrollo y mantenimiento de conductas resilientes, favoreciendo de este modo la calidad de vida (Jones, 2007; Vinaccia, Quiceno y Remor, 2012; Wahl et al., 2008; Walker et al., 2009). Situación que podría explicarse por el hecho de creer y tener fe en un ente superior que ayuda a vencer las vicisitudes que puedan presentarse a lo largo de la vida.

En este sentido, los creyentes considerarían que muchas de las circunstancias que se les presentan se encuentran en manos de su deidad, delegando en ella muchas de las preocupaciones que les atormentan. Cuando se estudia la relación entre las distintas dimensiones que conforman la resiliencia y la variable religión, encontramos que se alcanzan valores medios elevados en casi todas sus dimensiones, lo que corroborarían estudios como los de Camacho y Maldonado (2016), quienes destacan que la práctica de creencias y comportamientos religiosos actuaría como factor protector de la salud de las personas.

En consonancia con Arroyo (2014), quien señala la caída de la religión en términos generales, nuestros resultados muestran la existencia de una gran parte del alumnado que manifiesta ser ateo-agnóstico. Aun así, sigue siendo la religión cristiana la más profesada por el alumnado participante (Montes, 2016), seguida por la atea-agnóstica y la musulmana.

Por otro lado, algunos autores coinciden en que la mujer parece más resiliente que el hombre, refiriéndole cualidades y habilidades de interacción social para resolver y adaptarse positivamente al medio que la rodea, considerando incluso el género (femenino) como un factor de protección ante los factores de riesgos existentes y que pueden influir en la calidad de vida de los individuos (Álvarez y Cáceres, 2010; González, Valdez y Zabala, 2008; Prado y Águila, 2003); hecho controvertido, ya que otros autores, obtienen resultados que muestran puntuaciones más elevadas de resiliencia en los hombres (Fínez y Morán, 2015), existiendo también algunos estudios que no señalan diferencias significativas de género, como los de Gil, Orbea y Axpe (2012) y Zurita et al. (2016). 
En nuestro caso, el género femenino obtiene resultados más altos que el masculino en las dimensiones "Desafío de la conducta orientada a la acción" y "Espiritualidad", mientras que el hombre muestra un nivel superior en la dimensión "Optimismo y adaptación a situaciones estresantes", mientras que ambos géneros obtuvieron puntaciones similares en el resto de dimensiones resilientes. En este sentido, el estudio realizado por González y Valdez (2012), en cuanto al optimismo y pesimismo relacionado con la resiliencia, encontraron una diferencia significativa indicando un mayor pesimismo en las mujeres, lo que Seligman (2006) etiqueta como factor de riesgo para padecer depresión.

Al igual que otras investigaciones afines al presente estudio, la conformación de la muestra aplicada revela un alto porcentaje de participación femenina, lo que podría considerarse como una de las limitantes del trabajo, a tener en cuenta a la hora de interpretar los resultados (Edo-Gual, Tomás-Sábado, y Aradilla-Herrero, 2011; Schmidt-Riovalle et al., 2012). De hecho, ante la existencia de estudios contradictorios en relación con el género y la resiliencia, sería necesario continuar con trabajos de investigación empírica que aporten nuevos datos y conclusiones que apunten a uno u otro sentido.

En lo referente a la asociación entre resiliencia y la tendencia religiosa, los resultados mostraron diferencias en la dimensión "Desafío de la conducta orientada a la acción", en la que los universitarios musulmanes fueron los que presentaron una media inferior al resto de tendencias religiosas. No obstante, este colectivo obtuvo la mayor puntuación en la dimensión "Espiritualidad", sobre todo al compararlos con los ateo-agnósticos. Estos hallazgos pueden estar justificados por el estudio de Moyano y Trujillo (2014), quienes destacan cómo la comunidad musulmana muestra una mayor adhesión a su religión con respecto a la cristiana -que muestra una mayor división en la identidad religiosa. Asimismo, diversos estudios muestran cómo una mayor espiritualidad puede relacionarse con un mayor bienestar psicológico y social en ciertas religiones (Mensah, 2014; Moyano y Trujillo, 2014; Vinaccia et al., 2012).

Por último, resulta de interés destacar las limitaciones que presenta este estudio: la principal se adhiere al tipo de diseño - de tipo descriptivo y transversal, el cual no permite establecer relaciones causa-efecto; aunque resulta de gran utilidad para describir el estado de una cuestión. Otra limitación reside en la distribución de la muestra, pues los resultados podrían verse afectados por el mayor número de participantes de género femenino y tendencia cristiana. Del mismo modo, hubiera resultado de interés incluir otras variables de interés para este estudio, como son el rendimiento académico, la autoestima o el autoconcepto de los jóvenes.

Se puede concluir que la capacidad de resiliencia es un elemento esencial en la adaptación positiva del universitario, que parece tener diferencias en relación al género y tendencia religiosa. Por ello, investigar en el campo de la resiliencia y las variables que intervienen en ella resulta de gran utilidad para intervenir eficazmente en las situaciones que se presentan a lo largo de la vida del joven. Por tanto, se sugieren nuevos estudios que incluyan otras variables relacionadas para ampliar la visión del término resiliencia universitaria.

Como principales conclusiones podemos señalar:

- El estudio de la capacidad de resiliencia en la muestra revela una predicción donde la autoeficacia y resistencia al malestar constituye la dimensión que mejor puntúa en estudiantes universitarios, siendo la espiritualidad la que presenta menor relevancia.

- El análisis de la resiliencia en función del género reveló que el género masculino presentaba un mayor optimismo y adaptación a situaciones estresantes, mientras que el femenino se adhería a una mayor espiritualidad. Las diferencias en función de la tendencia religiosa mostraron que la tendencia cristiana y la ateo-agnóstica desafiaban mejor la conducta orientada a la acción y que la tendencia musulmana era la que mayor espiritualidad mostraba.

- El modelo de regresión logística binaria se ajustó de forma adecuada, aunque no predijo las diversas dimensiones de la capacidad de resiliencia en función del género. Si reveló que el pertenecer al género masculino aumentaba la probabilidad de realizar estudios de ciencias sociales así como de profesar una tendencia religiosa. 


\section{Referencias}

Allan, J. F., McKenna, J. y Dominey, S. (2014). Degrees of resilience: profiling psychological resilience and prospective academic achievement in university inductees. British Journal of Guidance y Counselling, 42(1), 9-25. doi:10.1080/03069885.2013.793784

Álvarez, R. y Cáceres, H. (2010). Resiliencia, rendimiento académico y variables sociodemográficas en estudiantes universitarios de Bucaramanga (Colombia). Psicología iberoamericana, 18(2), 37-46.

Álvaro, J. I., Zurita, F., Castro, M., Martínez, A. y García, S. (2016). Relación entre consumo de tabaco y alcohol y el autoconcepto en adolescentes españoles. Revista Complutense de Educación, 27(2), 533-550.

Arroyo, M. M. (2014). Interpretaciones sobre la situación del catolicismo y la secularización en España. Revista de pensamiento cristiano iglesia Viva, 257, 95-108.

Aslan, G. y Araza, A. (2015). Employee innovation resilience: a proposal for multidimensional construct. Business and Management Studies: An International Journal, 3(3), 290-308. doi:10.15295/bmij.v3i3.121

Bajaj, B. y Pande, N. (2016). Mediating role of resilience in the impact of mindfulness on life satisfaction and affect as indices of subjective well-being. Personality and Individual Differences, 93, 63-67.

doi:10.1016/j.paid.2015.09.005

Bandura, A. (2011). A social cognitive perspective on positive psychology. International Journal of Social Psychology, 26(1), 7-20. doi:10.1174/021347411794078444

Barrantes-Brais, K., Sánchez-Ureña, B. y Ureña-Bonilla, P. (2016). The effect of psychological and exercise interventions on college students' well-being and ill-being: a meta-analysis of randomized controlled trials. Journal of Sport and Health Research, 8(1), 65-82.

Beguerí, G., Klenzi, R. y Malberti, A. (abril, 2013). Determinación de factores resilientes en los alumnos universitarios. En C. Caluva, S. Aranguren y R. Muzachiodi (Comps.), XV Workshop de Investigadores en Ciencias de la Computación-wicc, 4, 1068-1072. Paraná, Argentina.

Bravo, H. y López, J. (2016). Resiliencia familiar: una revisión sobre artículos publicados en español. Revista de Investigación en Psicología, 18(2), 151-170. doi:10.15381/rinvp.v18i2.12089

Camacho, S. y Maldonado, E. (2016). El papel de la espiritualidad en la restauración de la salud. Revista Venezolana de Endocrinología y Metabolismo, 14(2), 93-95

Chacón, R., Castro, M., Caracuel, R., Padial, R., Collado, D. y Zurita, F. (2016). Perfiles de consumo de alcohol y tabaco en adolescentes andaluces de primer ciclo de educación secundaria. Health and Addictions Journal, 16(2), 93-104.

Chacón, R., Zurita, F., Martínez, A., Castro, M., Espejo, T. y Pinel, C. (2017). Relación entre factores académicos y consumo de videojuegos en universitarios. Un modelo de regresión. Pixel-Bits. Revista de Medios y Comunicación, 50, 109-121. doi: 10.12795/pixelbit.2017.i50.07

Connor, K. M. y Davidson, J. R. (2003). Development of a new resilience scale: the Connor Davidson resilience scale (CD-RISC). Depression and Anxiety, 18(2), 76-82. doi:10.1002/da.10113/full

De Barbieri, T. (1996). Certezas y malos entendidos sobre la categoría de género. Estudios básicos de Derechos Humanos, 4, 49-83. 
De la Fuente, J., López, M., Zapata, L., Martínez-Vicente, J. M., Vera, M. M., Solinas, G. y Fadda, S. (2014). Competencia para estudiar y aprender en contextos estresantes: Fundamentos de la utilidad eAfrontamiento del estrés académico. Electronic Journal of Research in Educational Psycology, 12(3), 717746. doi:10.14204/ejrep.34.14034

Del Rincón, I. B. (2016). Resiliencia y educación social. Revista Ibero-americana de Educação, 70(2), 79-94.

Edo-Gual, M., Tomás-Sábado, J. y Aradilla-Herrero, A. (2011). Miedo a la muerte en estudiantes de enfermería. Enfermería Clínica, 21(3), 129-135. doi:10.1016/j.enfcli.2011.01.007

Fínez, M. J. y Morán, M. C. (2015). La resiliencia y su relación con salud y ansiedad en estudiantes españoles. Una nueva visión de la Psicología: Psicología Positiva. International Journal of Developmental and Educational Psychology ,1(1), 409-416. doi:10.17060/ijodaep.2015.n1.v1.90

García del Castillo, J., García del Castillo-López, A., López-Sánchez, C. y Dias, P. C. (2016). Conceptualización de la resiliencia psicosocial y su relación con la salud. Health and Addictions, 16(1), 5968.

García-Portilla, M. P., Bascarán, M. T., Sáiz, P. A., Parellada, M., Bousoño, M. y Bobes, J. (2008). Banco de Instrumentos básicos para la práctica de la psiquiatría clínica (5a. ed.). Barcelona: Ars Médica.

Gil, I., Orbea, J. M., y Axpe, I. (2012). Aproximación al perfil resiliente de los/as futuros/as educadores/as sociales. Revista Internacional de Ciencias Sociales, 1(1), 1-15.

González, N. y Valdez, J. (2007). Resiliencia en niños. Psicología Iberoamericana, 15(2), 38-50.

González, N. y Valdez, J. (2012). Optimismo-pesimismo y resiliencia en adolescentes de una universidad pública. Ciencia Ergo Sum, 19(3), 207-214.

González, N., Valdez, J. y Zabala, Y. (2008). Resiliencia en adolescentes mexicanos. Enseñanza $e$ Investigación en Psicología, 13(1), 41-52.

González, N., Valdez, J., Van Barneveld, H. y González, S. (2012). Resiliencia y factores protectores en menores infractores y en situación de calle. Psicología y Salud, 22(1), 49-62.

Griera, M., Martínez-Ariño, J. A., Clot-Garrell, A. y García-Romeral, G. (2015). Religión e instituciones públicas en España. Hospitales y prisiones en perspectiva comparada. Revista Internacional de Sociología, 73(3), 1-13. doi:10.3989/ris.2015.73.3.e020

Hande, Z., Börkan, B., Erkman, F. y Serbest, S. (2016). Resilience as a mediator between parental acceptance-rejection and depressive symptoms among University students in Turkey. Journal of Counseling y Development, 94(2), 195-209. doi:10.1002/jcad.12076

Hu, T., Zhang, D. y Wang, J. (2015). A meta-analysis of the trait resilience and mental health. Personality and Individual Differences, 76, 18-27. doi:10.1016/j.paid.2014.11.039

Jones, J. (2007). Exposure to chronic community violence: resilience in african american children. Journal of Black Psychology, 33(2), 125-149. doi:10.1177/0095798407299511

Kalawski, J. y Haz, A. (2003). Y... ¿Dónde está la resiliencia? Una reflexión conceptual. Interamerican Journal of Psychology, 37(2), 365-372.

Llanos, M. (2009). Resiliencia y género. Promotoras comunitarias en Asia y América Latina. Temática Psicológica, 5(1), 51-58. 
López-Medina, M. L. (2006). Convivencia en Melilla: sus cuatro culturas. En E. Soriano, J. González y M. M. Osorio (Coords.), Convivencia y Mediación Intercultural (pp. 79-85). España: Universidad de Almería.

Losada, A. y Latour, M. I. (2012). Resiliencia. Contextualización e investigaciones en Argentina. Psiencia. Revista Latinoamericana de Ciencias Psicológicas, 4(2), 84-97. doi:10.5872/psiencia.v4i2.93

Maltby, J., Day, L., Żemojtel-Piotrowska, M., Piotrowski, J., Hitokoto, H., Baran, T. ... Flowe, H. (2016). An ecological systems model of trait resilience: cross-cultural and clinical relevance. Personality and Individual Differences, 98, 96-101. doi: 10.1016/j.paid.2016.03.100

Masten, A. (2014). Invited commentary: resilience and positive youth development frame works in developmental science. Journal of Youth and Adolescence, 43(6), 1018-1024.

doi:10.1007/s10964-014-0118-7

Mensah, Y. M. (2014). An analysis of the effect of culture and religion on perceived corruption in a global context. Journal of Bussiness Ethics, 121(2), 255-282. doi:10.1007/s10551-013-1696-0

Montes, M. A. (2016). ¿Por qué y cómo investigar las minorías religiosas? Universitas, 13(23), 125-141. doi:10.17163/uni.n23.23.2015.06

Moyano, M. y Trujillo, H. (2014). Intención de activismo y radicalismo de jóvenes musulmanes y cristianos residentes en un barrio marginal de una ciudad española. International Journal of Social Psichology, 29(1), 90-120. doi:10.1080/02134748.2013.878571

Obradors-Rial, N., Ariza, C. y Muntaner, C. (2014). Consumo de riesgo de alcohol y factores asociados en adolescentes de 15 a 16 años de la Cataluña Central: diferencias entre ámbito rural y urbano. Gaceta Sanitaria, 28(5), 381-385. doi:10.1016/j.gaceta.2014.04.004

Pérez-Agote, A. (2012). Cambio religioso en España: los avatares de la secularización. Madrid: Centro de Investigaciones Sociológicas.

Pisarska, A., Eisman, A., Ostaszewski, K. y Zimmerman, M. A. (2016). Alcohol and cigarette use among warsaw adolescents: factors associated with risk and resilience. Substance Use y Misuse, 51(10), 1-14. doi:10.3109/10826084.2016.1168442

Prado, R. y Águila, C. M. (2003). Diferencia en la resiliencia según género y nivel socioeconómico en adolescentes. Persona: Revista de la Facultad de Psicología, 6, 179-196.

Quiceno, J., Mateus, J., Cardenas, M., Villareal, D. y Vinaccia, S. (2013). Calidad de vida, resiliencia e ideación suicida en adolescentes víctimas de abuso sexual. Revista de Psicopatología y Psicología Clínica, 18(2), 107-117. doi:10.5944/rрpc.vol.18.num.2.2013.12767

Rehm, M. y Allison, B. (2009). Exploring spirituality of University FCS students: a resource for resiliency. Journal of Family and Consumer Sciences, 101(4), 12-17.

Rodríguez-Fernández, A., Ramos-Díaz, E., Ros, I., Fernández-Zabala, A. y Revuelta, L. (2016). Bienestar subjetivo en la adolescencia: el papel de la resiliencia, el autoconcepto y el apoyo social percibido. Suma psicológica, 23(1), 60-69. doi:10.1016/j.sumpsi.2016.02.002

Saavedra, G. E., Castro, R. A. y Saavedra, C. P. (2012). Autopercepción de los jóvenes universitarios y resiliencia: construcción de sus particularidades. Katharsis, 14, 89-105. doi:10.25057/25005731.450

Sánchez-Teruel, D. y Robles-Bello, M. A. (2014). Factores protectores que promueven la resiliencia ante el suicidio en adolescentes y jóvenes. Papeles del psicólogo, 35(2), 181-192. 
Sandoval, F., García-Jiménez, H. y Pérez-Mayo, A. (2015). Género y resiliencia ante la catástrofe ambiental. Revista Iberoamericana de Producción Académica y Gestión Educativa, 2(4), 1-20.

Satici, S. A. (2016). Psychological vulnerability, resilience, and subjective well-being: the mediating role of hope. Personality and Individual Differences, 102, 68-73. doi:10.1016/j.paid.2016.06.057

Seligman, M. (2006). La auténtica felicidad. España: Byblos.

Schmidt-Riovalle, J., Montoya-Juárez, R., Campos-Calderón C., García-Caro, M. P., Prados-Peña, D. y CruzQuintana, F. (2012). Efectos de un programa de formación en cuidados paliativos sobre el afrontamiento de la muerte. Revista Medicina Paliativa, 19(3), 113-20. doi:10.1016/j.medipa.2010.11.001

Simkin, H. y Azzollini, S. (2015). Personalidad, autoestima, espiritualidad y religiosidad desde el modelo y la teoría de los cinco factores. PSIENCIA. Revista Latinoamericana de Ciencia Psicológica, 7(2), 339-361. doi:10.5872/psiencia/7.2.61

Villacieros, I. (2016). La Resiliencia en los contextos de Refugio. Revista de Psicoterapia, 27(105), 139-155. doi: 10.33898/rdp.v27i105.129

Vinaccia, E., Quiceno, J. y Remor, E. (2012). Resiliencia, percepción de enfermedad, creencias y afrontamiento espiritual-religioso en relación con la calidad de vida relacionada con la salud en enfermos crónicos colombianos. Anales de Psicología, 28(2), 366-377. doi:10.6018/analesps.28.2.148821

Wahl, R., Cotton, S. y Harrison-Monroe, P. (2008). Spirituality, adolescent suicide, and the juvenile justice system. Southern Medical Journal, 101(7), 711-715. doi:10.1097/SMJ.0b013e31817a7e73

Walker, D., Reid, H., O'Neill, T. y Brown, L. (2009). Changes in personal religion/spirituality during and after childhood abuse: a review and synthesis. Psychological Trauma: Theory, Research, Practice, and Policy, 1(2), 130-145. doi:10.1037/a0016211

Zurita, F., Espejo, T., Cofré, C., Martínez, A., Castro, M. y Chacón, R. (2016). Influencia de la actividad física sobre la resiliencia en adultos con dolor de hombro. Sport TK-Revista Euroamericana de Ciencias del Deporte, 5(2), 53-58. doi:10.6018/264651

Zurita, Y., Castro, M., Linares, M. y Chacón, R. (2017). Resiliencia un elemento de prevención en actividad física. Sportis, Scientific Technical Journal of school Sport, Physical Education and Psychomotricity, 3(1), 564576. doi:10.17979/sportis.2017.3.1.1726 